\title{
The yield of immediate post lung biopsy $C T$ in predicting iatrogenic pneumothorax
}

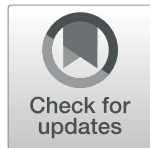

Rafael Y. Brzezinski ${ }^{1,2,3}$, Ifat Vigiser ${ }^{4}$, Irina Fomin ${ }^{5}$, Lilach Israeli ${ }^{5}$, Shani Shenhar-Tsarfaty ${ }^{1}$ and Amir Bar-Shai ${ }^{5^{*}}$ (D)

\begin{abstract}
Background: The most prevalent complication of percutaneous lung biopsy is pneumothorax (PNX). A routine immediate post-procedure CT scan (ICT) to spot PNX is done in many centers. However, the diagnostic yield of this practice has not been studied broadly. We sought to evaluate whether an ICT could replace the routine follow-up chest X-ray (CXR) in detecting procedure related PNX.

Methods: We examined case-records of 453 patients who underwent lung biopsy at our medical center. We analyzed findings from CXR performed 2-h after biopsy and from CT images at the site of biopsy acquired immediately after the procedure (ICT). Multivariate analysis was used to identify the risk factors for PNX, and we examined the concordance between ICT and CXR-2-h post-procedure.
\end{abstract}

Results: A total of 87 patients (19\%) were diagnosed with PNX on CXR-2-h post-procedure. ICT detected $80.5 \%$ of diagnosed PNX $(p<0.01)$. However, ICT demonstrated a negative predictive value of only 94\%, meaning 17 patients (6\%) with a negative ICT did eventually develop PNX seen on CXR. Furthermore, bleeding surrounding the puncture area spotted on ICT negatively predicted the development of PNX (OR $=0.495 \% \mathrm{Cl}$; 0.2-0.7).

Conclusions: We conclude that a CT scan performed immediately after percutaneous lung biopsy cannot replace the routine follow-up CXR in predicting iatrogenic PNX. Bleeding in the needle's tract may lower the risk for procedure-related PNX.

Keywords: Biopsy, Complications, Computed tomography, Lung cancer, Pneumothorax

\section{Background}

Computed tomography (CT) - guided percutaneous needle biopsy is a safe and precise method for the diagnosis of different pathologies in the thorax $[1,2]$.

The most prevalent complication of this procedure is pneumothorax (PNX), of which the reported prevalence ranges between 15 and 29\% [3]. PNX can lead to a considerable reduction in the patient's health state and longer hospitalization periods [4].

Reports regarding the risk factors and significant predictors of PNX are contradictory. A recent review highlighted lesion size, lesion depth and the presence of emphysema

\footnotetext{
* Correspondence: amirb@bmc.gov.il

${ }^{5}$ Division of Pulmonary Medicine, Barzilai Medical Center, Faculty of Health Sciences, Ben-Gurion University, 2 Hahistadrut Street, Ashkelon, Israel

Full list of author information is available at the end of the article
}

as significant risk factors for PNX [4], while other reports focus on the patient's posture during the procedure [5] or the degree of emphysema seen on CT [6].

A chest X-ray (CXR) performed $1-4 \mathrm{~h}$ after the procedure is performed in order to diagnose biopsy-related iatrogenic PNX [7-11]. And yet, a routine immediate post-procedure CT scan (ICT) to identify PNX is also done in many centers. The question of whether an ICT could replace the routine CXR performed during followup in diagnosing iatrogenic PNX has not been studied in a large sample of patients.

Therefore, we sought to examine the diagnostic yield of ICT in identifying iatrogenic PNX diagnosed on the follow-up CXR after lung biopsy. We aimed to evaluate the specific risk factors for PNX present in our cohort and to determine the characteristics of patients with 
undetected PNX on ICT that was detected only on CXR. The ultimate goal was to assess whether an ICT can replace the follow-up CXR in patients undergoing percutaneous lung biopsy.

\section{Methods}

\section{Study design}

We retrospectively examined case-records of 453 patients (mean age $69 \mathrm{yr}, 55 \%$ male) who underwent percutaneous lung biopsy in the Department of Pulmonary Diseases at our medical center. We reviewed the patients' imaging studies (chest CT results acquired immediately after the procedure (referred to as ICT) and CXR performed 2-h after biopsy). We collected demographic data as well as relevant medical history such as smoking history and the presence of emphysema.

The study was approved by the local ethics committee, conformed to the principles outlined in the Declaration of Helsinki, and Informed consent was waived due to the retrospective nature of the study.

\section{Percutaneous lung biopsy and image analysis}

All patients underwent CT-guided percutaneous lung biopsy in accordance with common procedure guidelines $[10,11]$. We performed core biopsies with an 18-gauge needle without the use of a coaxial needle. Patients did not receive any conscious sedation during the procedure. We categorized patients according to the performing physician. A total of 7 pulmonary specialists and 1 chest radiology specialist participated in our study. All performing physicians had at least 3 years of experience in performing lung biopsies. The number of punctures was documented for each technique as wells as the patient's posture during the procedure (i.e. decubitus, supine and prone). Biopsy samples were examined by a certified pathologist and were defined as malignant/benign/nondiagnostic.

An ICT was done immediately after the removal of the needle in order to document potential immediate complications such as PNX or bleeding. The ICT included CT images at the site of the biopsy. The patients were observed for 2-h at the vicinity of the procedure room. CXR was performed if any clinical deterioration was present or after completion of the 2-h observation period. Any clinical complication was treated immediately at the procedure site.

ICT scans and CXRs underwent manual surveys by trained pulmonologists at our medical center. We measured the lesions' size in both length and width, their location and distance from pleura, and documented the presence of bleeding surrounding the puncture area. A lesion's location was defined as being present at the upper lobes (both left and right) vs. lower lobes (including right middle lobe).

\section{PNX diagnosis}

In our institution, the follow-up CXR is routinely obtained $2 \mathrm{~h}$ after biopsy in order to assess procedurerelated PNX. Patients were categorized into two groups according to the presence of PNX on CXR. The need for chest tube insertion was documented for all patients diagnosed with PNX.

\section{Statistical analysis}

All continuous variables are displayed as mean \pm SD for normally distributed variables or median [interquartile range] for variables with abnormal distribution. Categorical variables are displayed as numbers (\%) of subjects within each group. The different characteristics in patients diagnosed with and without PNX were compared by a Student's $t$-test for normally distributed variables and by the Mann-Whitney $U$ test for non-normally distributed ones. To assess associations among categorical variables, we used a chi-square test. We measured sensitivity and specificity values for the ability of ICT to detect PNX present on CXR 2-h after the procedure.

To isolate significant risk factors for the development of PNX, we performed multivariate logistic regression to

Table 1 Patient-Characteristics According to Diagnosed Pneumothorax on Chest X-ray 2-h Post-Lung Biopsy

\begin{tabular}{|c|c|c|}
\hline Characteristic & Negative CXR & PNX on CXR \\
\hline Number of subjects & $366(81 \%)$ & $87(19 \%)$ \\
\hline Age, years & $70 \pm 13$ & $68 \pm 13$ \\
\hline Gender (Male) & $198(54 \%)$ & $53(61 \%)$ \\
\hline History of Smoking & $220(60 \%)$ & $42(48 \%)$ \\
\hline Pack Years & $17 \pm 30$ & $25 \pm 32$ \\
\hline Emphysema Diagnosis & $30(8 \%)$ & $8(9 \%)$ \\
\hline Lesion length, $\mathrm{cm}$ & $2.9 \pm 1.9$ & $2.1 \pm 1.4$ \\
\hline Lesion width, $\mathrm{cm}$ & $2.5 \pm 2$ & $1.8 \pm 1.2$ \\
\hline Distance from pleura, $\mathrm{cm}$ & $1.7 \pm 3.4$ & $2.7 \pm 1.8$ \\
\hline \multicolumn{3}{|l|}{ Lesion Locus } \\
\hline Upper Lobes & $158(43 \%)$ & $43(49 \%)$ \\
\hline Lower Lobes & $209(57 \%)$ & $44(51 \%)$ \\
\hline Number of punctures performed & $1.7 \pm 0.6$ & $1.5 \pm 0.6$ \\
\hline \multicolumn{3}{|l|}{ Patient Posture } \\
\hline Prone & $221(60 \%)$ & $54(62 \%$ \\
\hline Supine & $118(32 \%)$ & $21(24 \%)$ \\
\hline Decubitus & $27(8 \%)$ & $12(14 \%)$ \\
\hline Bleeding observed & $236(64 \%)$ & $53(60 \%)$ \\
\hline \multicolumn{3}{|l|}{ Pathologic Diagnosis } \\
\hline Non-Diagnostic & $19(5 \%)$ & $7(8 \%)$ \\
\hline Benign & $131(36 \%)$ & $39(45 \%)$ \\
\hline Malignant & 217 (59\%) & 41 (47\%) \\
\hline
\end{tabular}

Data presented as mean \pm SD or N (\%). CXR-Chest X-ray; PNX-Pneumothorax 
predict the presence of PNX on CXR. The model was adjusted for the following covariates: age, gender, smoking status, number of pack-years, lesion length and width, lesion distance from the pleura, categorized location of the lesion, performing physician, number of punctures performed, patient posture, pathologic diagnosis and the presence of bleeding on ICT. The variables included in our multivariate analysis were the ones that demonstrated statistical significance on univariate analysis, along with additional risk factors for iatrogenic PNX highlighted in past reports [4-6].

We performed one-way Analysis of Variance (ANOVA) with Tukey's test for multiple comparisons to compare characteristics between the four different groups of patients according to ICT results; false/true negative and false/true positive test results. $P$-values of $<0.05$ were considered statistically significant.

We used the IBM SPSS Statistics 22.0 statistical package (IBM Corporation, Armonk, New York, USA) and GraphPad Prism version 7.00 (GraphPad Software, La Jolla, CA, USA) for all statistical analysis.

\section{Results}

\section{Study population}

A total of 87 patients (19\%) were diagnosed with PNX on CXR performed 2-h post-procedure. Twenty-five patients required chest tube insertion accounting for $29 \%$ of PNXs and 5.5\% of all lung-biopsies. Patients' characteristics are shown in Table 1. Patients diagnosed with PNX presented with smaller lesions in both length and width, as well as a more distant location from the pleura (Table 1). Notably, patients with and without PNX demonstrated a similar prevalence of existing comorbidities such as emphysema and smoking history. Men had slightly higher rates of PNX compared to women, albeit not statistically significant; $61 \%$ vs $54 \%$, $(p=0.15)$. The number of needle passes during the procedure was slightly higher in patients without PNX; $1.7 \pm 0.6$ vs. $1.5 \pm 0.6,(p=0.02)$.

\section{Detected bleeding on ICT lowered the risk for PNX}

We first sought to evaluate the risk factors for developing PNX in our cohort (Fig. 1). In-line with previous reports [3, 4], multivariate analysis showed that the lesion's distance from the pleura predicted higher rates of PNX (OR=1.6, 95\% CI; 1.4-1.9). However, the lesion's size, categorized location, number of needle passes, and the patient's posture during the procedure were not significant predictors of PNX in our cohort (Fig. 1). The pathologic diagnosis and the performing physician were not significant predictors as well.

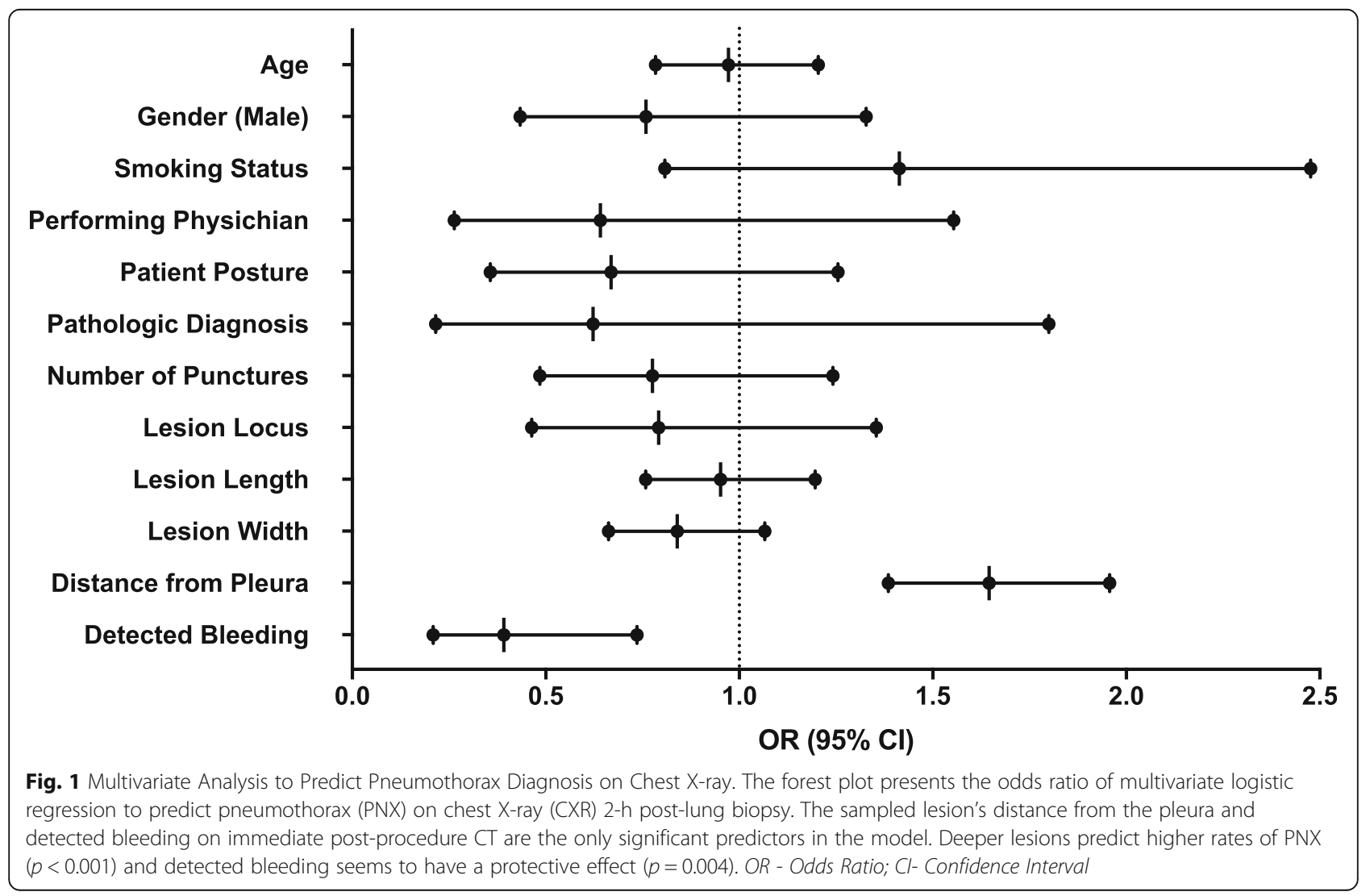




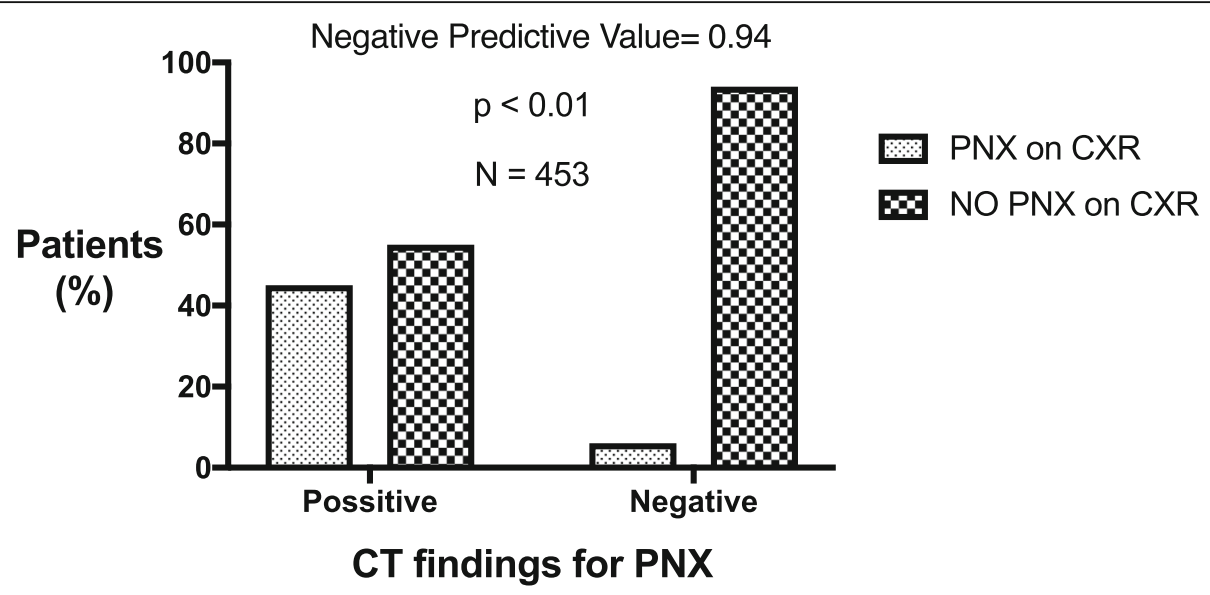

Fig. 2 The Yield of Post-Lung Biopsy CT in Predicting Pneumothorax on Chest X-Ray 2-h Post-Procedure. The clustered bar chart shows the percentage of patients diagnosed with pneumothorax (PNX) on Chest X-ray 2-h post-procedure within patients diagnosed with ('Positive') and without ('Negative') PNX on immediate CT. P-value was calculated by a chi-square test. CT-computed tomography; PNX-Pneumothorax

Table 2 Characteristics of Patients with a Negative CT Post- Lung Biopsy According to Diagnosed Pneumothorax on Follow-up Chest X-ray

\begin{tabular}{|c|c|c|c|}
\hline Characteristic & No PNX on CXR & PNX on CXR & $\boldsymbol{P}$-value \\
\hline Number of subjects & 282 & 17 & \\
\hline Age, years & $69 \pm 13$ & $61 \pm 14$ & 0.02 \\
\hline Gender (Male) & $150(53 \%)$ & $10(59 \%)$ & 0.8 \\
\hline History of Smoking & $113(40 \%)$ & $6(35.3 \%)$ & 0.8 \\
\hline Pack Years & $18 \pm 31$ & $17 \pm 28$ & 0.86 \\
\hline Emphysema Diagnosis & $20(7 \%)$ & 0 & 0.6 \\
\hline Lesion length, $\mathrm{cm}$ & $3.1 \pm 2.1$ & $2 \pm 1.1$ & $<0.01$ \\
\hline Lesion width, $\mathrm{cm}$ & $2.8 \pm 2.2$ & $1.6 \pm 0.8$ & $<0.01$ \\
\hline Distance from pleura, cm & $1.6 \pm 3.8$ & $3.2 \pm 1.8$ & $<0.01$ \\
\hline \multicolumn{4}{|l|}{ Lesion Locus } \\
\hline Upper Lobes & $117(42 \%)$ & $11(65 \%)$ & \multirow[t]{2}{*}{0.06} \\
\hline Lower Lobes & $165(58 \%)$ & $6(35 \%)$ & \\
\hline Number of punctures performed & $1.8 \pm 0.7$ & $1.5 \pm 0.5$ & 0.16 \\
\hline \multicolumn{4}{|l|}{ Patient Posture } \\
\hline Prone & $168(60 \%)$ & $11(65 \%)$ & \multirow[t]{3}{*}{0.01} \\
\hline Supine & $96(34 \%)$ & $2(12 \%)$ & \\
\hline Decubitus & $18(6 \%)$ & $4(23 \%)$ & \\
\hline \multicolumn{4}{|l|}{ On-spot Pathologic Diagnosis } \\
\hline Non-Diagnostic & $15(5 \%)$ & $3(18 \%)$ & \multirow[t]{3}{*}{0.11} \\
\hline Benign & $102(36 \%)$ & $6(35 \%)$ & \\
\hline Malignant & 165 (58\%) & $8(47 \%)$ & \\
\hline
\end{tabular}

$P$-values $<0.05$ are shown in bold

Data presented as mean \pm SD or N (\%). CXR-Chest X-ray; PNX-Pneumothorax 
Finally, our analysis demonstrated that bleeding surrounding the puncture area spotted on ICT negatively predicted the development of PNX on CXR (OR $=0.4$ 95\% CI; 0.2-0.7, $p=0.004$ ) (Fig. 1).

\section{ICT did not detect all PNX seen on CXR}

We next sought to determine the diagnostic yield of ICT in detecting iatrogenic PNX. ICT detected $80.5 \%$, of patients diagnosed with PNX on CXR $(p<0.01)$. More importantly, ICT demonstrated a negative predictive value of only $94 \%$, meaning 17 patients $(6 \%)$ with a negative ICT did eventually develop PNX seen on CXR 2-h postprocedure (Fig. 2).

ICT identified 85 patients (23\%) with PNX that did not develop into a significant PNX identified on CXR, accounting for a specificity rate of $77 \%$ for ICT in predicting PNX.

A total of 22 patients that had PNX spotted on ICT required chest tube drainage $(14.2 \%$ of positive ICT patients). Three more patients with a negative ICT (1\% of negative ICT patients) also required a chest tube insertion.

\section{Miss-diagnosed PNX on ICT related to smaller and more distant lesions}

Finally, we aimed to characterize the sub-group of patients (6\%) that had a diagnosed PNX on CXR that was not detected on ICT (Table 2). These patients presented with smaller lesions in both length and width, as well as a more distant location from the pleura (Fig. 3). Of note, this sub-group of patients were relatively younger than patients with PNX spotted on both ICT and CXR; $61 \pm$ 14 vs. $69 \pm 13(p=0.02)$ (Table 2, Fig. 3).

\section{Discussion}

The main finding of this study is that an ICT cannot replace a CXR performed 2-h post-lung biopsy in detecting iatrogenic PNX. In this single-center experience, ICT detected $80 \%$ of PNX seen on the follow-up CXR with a $6 \%$ rate of false negative test results. Approximately $1 \%$
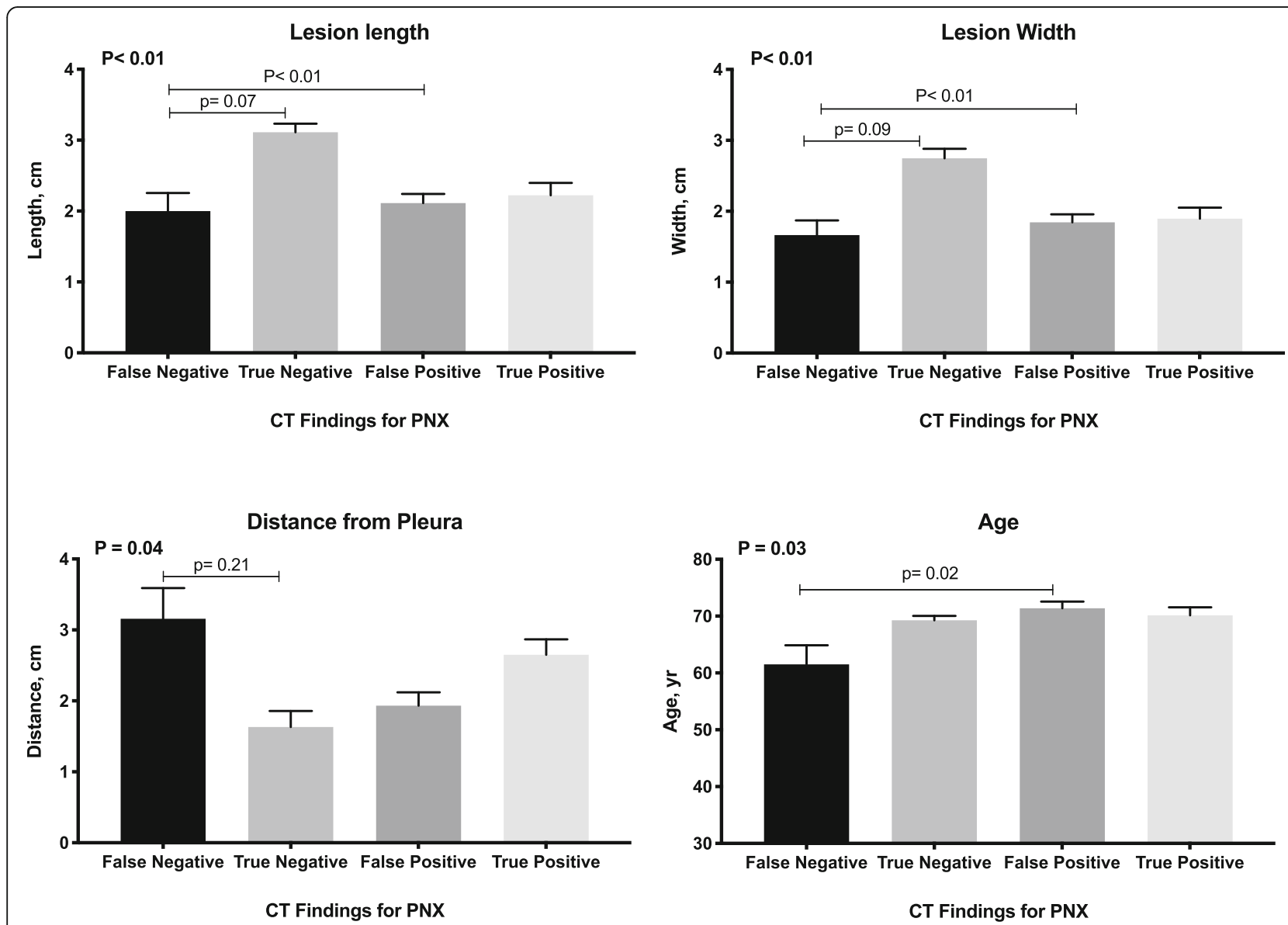

Fig. 3 Characteristics of Lesions According to Post Lung Biopsy CT results. We categorized the cohort according to test results of the immediate post lung biopsy $C T$ in predicting pneumothorax on chest X-ray. Patients with a false negative test result had smaller lesions in both length (upper left), width (upper right), were deeper within the lung (lower left) and younger in age (lower right). Data presented as mean \pm SEM. Pvalues were calculated by one-way ANOVA followed by Tukey's test for multiple comparisons. CT- Computed Tomography; PNX- Pneumothorax 
of patients with a negative ICT ended up requiring a chest tube insertion. PNX is a life-threatening complication and this diagnostic yield does not support waiving the follow-up CXR in detecting PNX.

Our findings are in-line with a recent report by Lim et al. [12] that depicted the time-dependent incidence of PNX after lung biopsy. Similar to our findings, an ICT detected $81 \%$ of PNX seen on CXR 4-h post procedure. Lim et al. reported an even higher rate of false negative results $(\sim 18 \%)$ and this might be due to the longer follow-up period in their study (4 vs $2 \mathrm{~h}$ post procedure).

The incidence of PNX in our cohort (19\%) is similar to previous reports regarding lung biopsy complication rates recently reviewed [3]. The significant risk factors for this complication vary across different study cohorts $[4-6,8,13]$. Our multivariate analysis shows that the distance of the sampled lesion from the pleura is the main risk factor for PNX. This is in accordance with the majority of past reports $[3,4,11]$. However, lesion size or patient posture were not significant risk factors in our cohort.

Our findings show, for the first time, that detected bleeding on ICT was protective against the development of PNX (OR $=0.4$ 95\% CI; 0.2-0.7, $p=0.004)$. This concept has been discussed previously (personal communication) but did not have sufficient supporting evidence until now. We hypothesize that processes associated with blood clot formation at the puncture-area affect the visceral pleura and might aid in preventing air leak from the lung parenchyma to the pleural space. Future more mechanistic-oriented studies aimed to test this theory are warranted. Lim et al. reported a similar protective effect for the presence of hemoptysis in the development of PNX ( $\mathrm{HR}=0.503 ; \mathrm{CI}=0.355-0.713)$ [12]. Detected bleeding on ICT is seen earlier and could, in some cases, lead to hemoptysis on follow-up. Their findings provide further support for our theory.

Our study has several limiting aspects. Primarily, this is a single center retrospective experience and is hence subject to residual confounding effects. And yet, our major complication rates are in-line with previous reports and thus provide support to the external validity of our findings. Furthermore, this study was not designed to include the effect of final biopsy results nor the diagnostic yield of the lung biopsy itself. However, the pathologic results did not show significant correlations between the type of sampled lesion and the risk for PNX. Finally, future studies should include a quantitative assessment of the size of spotted PNXs in both CXR and ICT.

The future clinical implication of our findings is debatable. Our study demonstrates the limited yield of ICT in predicting one of the major complications of percutaneous lung biopsy. In light of the immediate high risk of PNX in this clinical scenario, it does not seem possible to perform an ICT without also obtaining a CXR 2-h after the procedure. However, completely avoiding ICT is also inconceivable since ICT aids to detect other major and life-threatening complications (e.g. massive bleeding and tension PNX). Future prospective studies are warranted to better determine the ultimate time point to perform the first follow-up CXR after lung biopsy.

\section{Conclusions}

A CT scan performed immediately after lung biopsy cannot replace a routine chest X-ray 2-h post-procedure in assessing iatrogenic pneumothorax. Bleeding in the needle's tract may lower the risk for procedure-related pneumothorax.

\section{Abbreviations}

$\mathrm{CT}$ : Computed tomography; CXR: Chest X-ray; ICT: Immediate post-procedure CT scan; PNX: Pneumothorax

\section{Acknowledgments \\ None.}

\section{Authors' contributions}

$R Y B, A B$, and IV participated in study conception and design. AB, IV, IF, and LI performed the acquisition of data. RYB, SST, IV and AB participated in analysis and interpretation of data. RYB, IV, and $A B$ drafted the manuscript and IF, SST and LI helped in critical review of the manuscript. All of the authors have read and approved the final manuscript.

\section{Funding}

None.

\section{Availability of data and materials}

The datasets used and/or analyzed during the current study are available from the corresponding author on reasonable request.

\section{Ethics approval and consent to participate}

The study was approved by the Tel Aviv Medical Center Institutional Review Board (IRB), conformed to the principles outlined in the Declaration of Helsinki and Informed consent was waived due to the retrospective nature of the study.

\section{Consent for publication}

Not applicable.

\section{Competing interests}

The authors declare that they have no competing interests.

\section{Author details}

"Department of Internal Medicine "C", "D" and "E", Tel Aviv Sourasky Medical Center and Sackler Faculty of Medicine, Tel Aviv University, Tel Aviv, Israel. ${ }^{2}$ Neufeld Cardiac Research Institute, Sackler Faculty of Medicine, Tel Aviv University, Tel Aviv, Israel. ${ }^{3}$ Tamman Cardiovascular Research Institute, Leviev Heart Center, Sheba Medical Center, Tel-Hashomer, Israel. ${ }^{4}$ Department of Neurology, Tel Aviv Sourasky Medical Center and Sackler Faculty of Medicine, Tel Aviv University, Tel Aviv, Israel. ${ }^{5}$ Division of Pulmonary Medicine, Barzilai Medical Center, Faculty of Health Sciences, Ben-Gurion University, 2 Hahistadrut Street, Ashkelon, Israel.

Received: 10 October 2019 Accepted: 31 March 2020

Published online: 15 April 2020

References

1. Deng C-J, Dai F-Q, Qian K, Tan Q-Y, Wang R-W, Deng B, et al. Clinical updates of approaches for biopsy of pulmonary lesions based on systematic review. BMC Pulm Med. 2018;18:146. 
2. Anzidei M, Porfiri A, Andrani F, Di Martino M, Saba L, Catalano C, et al. Imaging-guided chest biopsies: techniques and clinical results. Insights Imaging. 2017;8:419-28.

3. Heerink WJ, de Bock GH, de Jonge GJ, Groen HJM, Vliegenthart R, Oudkerk M. Complication rates of CT-guided transthoracic lung biopsy: meta-analysis. Eur Radiol. 2017;27:138-48.

4. Boskovic T, Stanic J, Pena-Karan S, Zarogoulidis P, Drevelegas K, Katsikogiannis $\mathrm{N}$, et al. Pneumothorax after transthoracic needle biopsy of lung lesions under CT guidance. J Thorac Dis. 2014;6(Suppl 1):S99-107.

5. Nakamura M, Yoshizako T, Koyama S, Kitagaki H. Risk factors influencing chest tube placement among patients with pneumothorax because of $C T$ guided needle biopsy of the lung. J Med Imaging Radiat Oncol. 2011;55: 474-8.

6. Lendeckel D, Kromrey M-L, Ittermann T, Schäfer S, Mensel B, Kühn J-P. Pulmonary emphysema is a predictor of pneumothorax after $\mathrm{CT}$-guided transthoracic pulmonary biopsies of pulmonary nodules. PLoS One. 2017;12: e0178078.

7. Perlmutt LM, Braun SD, Newman GE, Oke EJ, Dunnick NR. Timing of chest film follow-up after transthoracic needle aspiration. Am J Roentgenol. 1986; 146:1049-50.

8. Choi CM, Um SW, Yoo CG, Young WK, Sung KH, Shim YS, et al. Incidence and risk factors of delayed pneumothorax after transthoracic needle biopsy of the lung. Chest. 2004;126:1516-21.

9. Taleb S, Jalaeian H, Frank N, Golzarian J, D'Souza D. Is a routine chest X-ray necessary in every patient after percutaneous CT-guided lung biopsy? A retrospective review of 278 cases. Cardiovasc Intervent Radiol. 2017:40: $1415-20$.

10. Manhire A, Charig M, Clelland C, Gleeson F, Miller R, Moss H, et al. Guidelines for radiologically guided lung biopsy. Thorax. 2003;58:920-36.

11. Birchard K. Transthoracic Needle Biopsy. Semin Intervent Radiol. 2011;28: 087-97.

12. Lim WH, Park CM, Yoon SH, Lim H, Hwang EJ, Lee JH, et al. Time-dependent analysis of incidence, risk factors and clinical significance of pneumothorax after percutaneous lung biopsy. Eur Radiol. 2018;28:1328-37.

13. Wang S, Tu J, Chen W. Development and validation of a prediction pneumothorax model in CT-guided transthoracic needle biopsy for solitary pulmonary nodule. Biomed Res Int. 2019;2019:1-7.

\section{Publisher's Note}

Springer Nature remains neutral with regard to jurisdictional claims in published maps and institutional affiliations.

Ready to submit your research? Choose BMC and benefit from:

- fast, convenient online submission

- thorough peer review by experienced researchers in your field

- rapid publication on acceptance

- support for research data, including large and complex data types

- gold Open Access which fosters wider collaboration and increased citations

- maximum visibility for your research: over $100 \mathrm{M}$ website views per year

At $\mathrm{BMC}$, research is always in progress.

Learn more biomedcentral.com/submissions 